\title{
A New Methodology for Studying the Equity Premium*
}

\author{
Elie Appelbaum ${ }^{\dagger}$ and Parantap Basu ${ }^{\ddagger}$
}

November 2003

\begin{abstract}
This paper provides a new framework for the derivation and estimation of consumption and the equity premium functions. The novelty in our approach is that it does not require the explicit specification of the underlying consumer preferences. Applying duality in a dynamic context, we derive simple explicit expressions for both consumption and equity premium. We show that equity premium and consumption functions can be easily obtained by "Roy's Identity like" equations from the indirect utility function.

Using aggregate US data (1929-2000) we estimate the consumption and equity premium functions using a nonparametric technique. We find that the model does well in explaining the observed smooth consumption patterns and does reasonably well in explaining the high mean and volatility of equity premia.
\end{abstract}

Keywords: Consumption, Equity Premium, Moments.

Address: Elie Appelbaum, Department of Economics, York University, 4700 Keele Street, Toronto, Ontario, M3J 1P3, Canada phone: 416-736-5083,

\footnotetext{
${ }^{*}$ We wish to thank J. Racine for his help with the nonparametric estimation. The second author gratefully acknowledges a Fordham research grant to sponsor this project. An earlier version of this paper was presented at the Australian Meetings of the Econometric Society, Sydney, 2003.

†York University.

$\ddagger$ Fordham University.
} 


\section{Introduction}

Following the seminal paper of Mehra and Prescott (1985), macroeconomists faced a serious challenge to solve, what has become known as, the equity premium puzzle. The challenge is to find a general equilibrium model which can replicate the observed smoothness of aggregate consumption, as well as the high mean and volatility of equity premia. ${ }^{1}$ Within the consumption capital asset pricing framework (known as consumption CAPM), several preference structures were proposed to solve this puzzle. ${ }^{2}$ These include: habit formation, time nonseparability and nonexpected utility. While these preference structures enrich our understanding and address some aspects of the puzzle, they often pose serious technical difficulties. One of the major challenges is tractability. As one enriches the preference structures, it becomes increasingly difficult to obtain a tractable solution for consumption and equity premium. On the other hand, when one can obtain closed form solutions for consumption and equity premium, the restrictions imposed by preferences are, often, rejected by the data. At this point, it is still a challenge to find a framework that: (i) allows for sufficiently general preferences; (ii) maintains the spirit of the consumption CAPM; (iii) is tractable; and (iv) can address the equity premium puzzle.

The purpose of this paper is twofold. First, we provide an alternative, more general and practically useful framework for the derivation of consumption and equity premium functions. Second, we implement the methodology empirically and use it to address the equity premium puzzle.

The theoretical idea is simple and is based on the methodology that is, by now, standard in the applications of duality in consumer and producer theories. Rather than focusing on the primal utility function, we focus on the dual indirect utility function (IUF, hereafter) and show that relationships of interest can be easily derived from this IUF. Specifically, we use principles of duality, applied in a dynamic context, to derive the consumption function and an explicit simple expression for the equity premium. It turns out that the equity premium can be easily obtained from the ratio of marginal utilities (obtained from the IUF) of the standard deviation and the mean of market returns. This implies that the Sharpe Ratio is easily obtained, within a general equilibrium setting, by the slope of the IUF, in mean/standard deviation space. This result is reminiscent of the standard result in mean variance portfolio choice theory. Similarly, the consumption function is easily obtained from the ratio of marginal utilities of means of market return and household income. These expressions provide simple estimable equations for equity premium and consumption functions, while being consistent with the underlying general equilibrium model.

Our approach has several advantages. First, by enabling us to derive an explicit and very general expression for the equity premium, it provides considerable flexibility in addressing the equity premium puzzle. Second, it is applicable to any underlying preference structure, including non-expected utility. ${ }^{3}$ Third, the expressions for the equity premium and

\footnotetext{
${ }^{1}$ The equity premium is calculated as the differeence between the return on a market portfolio (e.g., approximated by the $\mathrm{S} \& \mathrm{P}$ returns) and the return on a risk free bond (measured by the US T-bill rate). The work following the seminal paper by Mehra and Prescott (1985) tried to match the first moment of the equity premium. For the 1926-99 period, the equity premium averaged about 7\% according to Center for Research in Security Prices, and about 6\% according to Mehra and Prescott (1985), for the 1879-1979 period. Under reasonable assumptions, however, the standard asset pricing models predict an equity premium of only $0.25 \%$. Subsequently, Hansen and Jagannathan (1991), as well as Lettau and Uhlig (2002), emphasize the volatility component of the equity premium puzzle.

${ }^{2}$ Other possible explanations for the puzzle that were proposed in the literature include: market imperfections (Jagannathan, McGrattan and Scherbina, 2000, Treynor, 1994) and crash state (Rietz, 1988). See also Cochrane and Hansen (1992) and Kocherlakota (1996) for a literature review.

${ }^{3}$ The analysis of financial variables without committing to explicit preferences is becoming quite common. For example,
} 
Sharpe ratio come directly from the indirect utility function, without explicitly involving the preference based discount factor. ${ }^{4}$

We implement our approach empirically and use it to address the equity premium puzzle. We estimate the consumption and equity premium functions, with US data for the period 1929-2000. To avoid the difficulties involved in choosing a functional form for the IUF, we estimate the consumption and equity premium functions using a nonparametric estimation technique, thus "letting the data" choose the appropriate form. We find that the model explains the smooth consumption patterns very well. In addition, it performs reasonably well in explaining the high mean and volatility of equity premia. It appears, therefore, that our general framework (theoretically and empirically), allows us to make good progress in addressing and trying to resolve the equity premium puzzle.

The paper is organized as follows. The following section lays out the theoretical framework. Section 3 implements it empirically. Section 4 concludes.

\section{Theoretical Framework}

Consider a representative household who, at each date $t$,consumes $c_{t}$, supplies a fixed amount of labor (normalized at unity) to the firm, at a competitive wage, $\psi_{t}$, and participates in share and bond markets. A share is an ownership claim to the capital stock in the economy. Define the stock of shares held by the household at date $t$ as $z_{t}$. At date $t$ the household buys net shares of, $z_{t}-z_{t-1}$ at a price $p_{t}$, in the stock market. Each share yields a dividend, $d_{t}$. The household also buys and sells bonds, $b_{t}$, at a price $p_{t}^{b}$, in the bond market. Such a bond pays one unit of sure consumption in the following period.

The household, thus, faces the sequential budget constraint:

$$
z_{t-1} d_{t}+p_{t} z_{t-1}+b_{t-1}+\psi_{t}-T_{t}=c_{t}+p_{t} z_{t}+p_{t}^{b} b_{t}
$$

where $T_{t}$ is a lump-sum tax imposed by the government. ${ }^{5}$

The household's wealth at date $t$, denoted by $w_{t}$, is given by:

$$
w_{t}=z_{t-1} d_{t}+p_{t} z_{t-1}+b_{t-1}+\psi_{t}-T_{t}
$$

Using (1) one may rewrite the household's budget constraint as:

$$
w_{t+1}=\left(w_{t}-c_{t}\right)\left[R_{t+1} \lambda_{t}+R_{t}^{f}\left(1-\lambda_{t}\right)\right]+y_{t+1}
$$

where

$$
\begin{aligned}
R_{t+1} & \equiv \frac{p_{t+1}+d_{t+1}}{p_{t}} \\
R_{t}^{f} & \equiv \frac{1}{p_{t}^{b}} \\
y_{t+1} & =\psi_{t+1}-T_{t+1}
\end{aligned}
$$

Alvarez and Jermann (2000) use asset prices to measure the cost of business cycles without fully specifying consumer preferences.

${ }^{4}$ In the consumption CAPM literature, the equity premium and the resulting Sharpe ratio depend on the consumption based stochastic discount factor. Lettau and Uhlig (2002) show that one requires an implausibly large elasticity of this discount factor with respect to the consumption innovations. Similar problems are reported by Hansen and Jagannathan (1991), who derive an upper bound for the Sharpe ratio for asset returns. They show that one requires a highly variable stocahstic discount factor to meet this upper bound, which is not consistent with the observed smooth process for consumption.

${ }^{5} T_{t}$ is the lump-sum taxes net of transfer. We assume that governement spending is wasteful in the sense that it does not give houshold any direct utlity; nor does it serve any productive role in the economy. 
and $\lambda_{t}$ is the market portfolio share, defined as:

$$
\lambda_{t}=\frac{p_{t} z_{t}}{p_{t} z_{t}+p_{t}^{b} b_{t}}
$$

The household solves its decision problem facing the processes $\left\{R_{t}\right\},\left\{y_{t}\right\},\left\{R_{t}^{f}\right\}$ as (parametrically) given. We assume that at each point in time, $R_{t+1}$ and $y_{t+1}$ are continuous and bounded random variables, but $R_{t}^{f}$ is known. Let the evolution of $R_{t+1}$ and $y_{t+1}$ be given by the stationary transition function $G\left(R_{t+1}, y_{t+1} \mid R_{t}, y_{t}\right)$. For any $t$, define the moments of the distribution $G$ as $m_{t}$. Since the random variables are concentrated on a compact support, the moments exist and uniquely determine the distribution $G(.){ }^{6}$ For any $m_{t}$ there is, therefore, a unique distribution, denoted by $G^{m_{t}}$, whose moments are given by the same $m_{t}$.

Following Kreps and Porteus (1978), given wealth $w_{t}$, the joint distribution function, $G($.$) , and a continuous aggregator function, U$, the solution to the household's problem can be obtained by solving the following functional equation:

$$
\begin{gathered}
\phi\left(w_{t} ; R_{t}^{f}, G^{m_{t}}\right) \equiv \\
=\operatorname{Max}_{c_{t}, \lambda_{t}} U\left\{c_{t}, \int \phi\left[\left(w_{t}-c_{t}\right)\left[R_{t+1} \lambda_{t}+R_{t}^{f}\left(1-\lambda_{t}\right)\right]+y_{t+1}\right] d G^{m_{t}}\right\}
\end{gathered}
$$

where $\phi\left(w_{t} ; R_{t}^{f}, G^{m_{t}}\right)$ is the value functional that characterizes the solution to the household's problem.

Given the relationship between distributions and moments, we can define the value function as: ${ }^{7}$

$$
H\left(w_{t}, R_{t}^{f}, m_{t}\right) \equiv \phi\left(w_{t} ; R_{t}^{f}, G^{m_{t}}\right)
$$

The value function, $H\left(w_{t}, R_{t}^{f}, m_{t}\right)$, is an indirect utility function and can be used in the same way as in standard consumer theory. Moreover, $H\left(w_{t}, R_{t}^{f}, m_{t}\right)$ can be shown to be continuous and convex in $m$ if we have expected utility and it maintains some monotonicity properties. $^{8}$

Assuming an interior solution, the first order conditions for consumption, $c_{t}$, and the portfolio share, $\lambda_{t}$, are given by: ${ }^{9}$

$$
U_{2} E_{t}\left[M_{t+1} \widetilde{R}_{t+1}\right]=1
$$

and

$$
E_{t}\left[\frac{\partial \phi}{\partial w_{t+1}}\left(R_{t+1}-R_{t}^{f}\right)\right]=0
$$

\footnotetext{
${ }^{6}$ Bounded support is a sufficient condition for the distribution function to be uniquely determined by the moments. This is the so called "moments problem". See, for example, Wilks (1964), Theorem 5.5.1. p. 126, Kendall (1969), Corollary 4.22, p. 110.

${ }^{7}$ Since $c_{t} \in \mathcal{C}$ and $\lambda_{t} \in \Lambda$, non-empty and compact subsets in $\mathcal{R}_{+}^{1}$ and given that $F$ is continuous (and $E_{t}\left[\phi\left(w_{t+1} ; G\right)\right.$ is continuous in $G$ ), with a compact range, it follows from the Theorem of the Maximum (Berg (1963)), that $\phi$ is continuous and the optimal solution is upper semi-continuous. This, of course, is the standard result from consumer theory. See Kreps and Porteus (1978), Mas-Collel et. al. (1995). See also Machina (1984), where it is shown that, in the case of expected utility (in the context of a static model), convexity and continuity in the distribution completely characterize the indirect utility functional, so that any functional with these properties is the indirect utility functional for some preferences.

${ }^{8}$ If we do not have expected utility, $H\left(w_{t}, R_{t}^{f}, m_{t}\right)$ may be either convex, or concave in the moments. The proofs are available upon request.

${ }^{9}$ See Appendix A.I for derivation.
} 
where

$$
\widetilde{R}_{t+1} \equiv\left[R_{t+1} \lambda_{t}+R_{t}^{f}\left(1-\lambda_{t}\right)\right]
$$

$M_{t+1}$ is the stochastic discount factor, defined by:

$$
M_{t+1} \equiv \frac{\frac{\partial \phi}{\partial w_{t+1}}}{\frac{\partial \phi}{\partial w_{t}}}
$$

and $U_{2} \equiv \frac{\partial U}{\partial E_{t}\left[\phi\left(w_{t+1} ; G\right)\right]}$.

Since all households are identical, in equilibrium $b_{t}=0$ and it follows that: ${ }^{10}$

$$
\lambda_{t}=1
$$

which, in turn, implies that in equilibrium,

$$
\widetilde{R}_{t+1}=R_{t+1}
$$

and the constraint (2) becomes:

$$
w_{t+1}=\left(w_{t}-c_{t}\right) R_{t+1}+y_{t+1}
$$

Consequently, the first order conditions for consumption, (8) becomes:

$$
U_{2} E_{t}\left[M_{t+1} R_{t+1}\right]=1
$$

In principle, applying a primal approach, we could derive all underlying asset prices (returns), solve for optimal consumption and then compute the equity premium. However, it is very difficult to obtain closed form solutions for sufficiently general preferences. For simpler preferences, closed form solutions are possible, but the restrictions imposed in these cases are usually rejected by the data. ${ }^{11}$

In the next section, we propose an alternative method for the derivation of consumption and equity premium functions. This is a dual approach that uses the indirect utility function to obtain explicit expressions for consumption and equity premium for any underlying preferences. We show that consumption and equity premium are easily obtained as functions of the moments of the forcing processes (as well as wealth) and can, therefore, be easily estimated.

\subsection{Consumption Function}

One of the attractive features of our framework is that it enables us to derive the consumption and equity premium functions for any underlying preferences by applying standard duality principles. To see this, let the stochastic processes for $y_{t+1}, R_{t+1}$ be parameterized as:

$$
y_{t+1}=\bar{y}_{t}+\sigma_{y_{t}} \varepsilon_{y_{t+1}}
$$

\footnotetext{
${ }^{10}$ See Appendix B for a more detailed discussion of the economy's equilibrium.

${ }^{11}$ See Lettau and Uhlig (2002) for an excellent survey of various types of closed form solutions for equity premium functions and their limitations.
} 


$$
R_{t+1}=\bar{R}_{t}+\sigma_{R_{t}} \varepsilon_{R_{t+1}}
$$

where $\varepsilon_{R_{t+1}}, \varepsilon_{y_{t+1}}$ are white noise random variables (with zero mean and variance of one). We also assume that the above processes satisfy stationarity conditions.

Using (16) and (17), the budget constraint is given by:

$$
w_{t+1}=\left(w_{t}-c_{t}\right)\left\{\left[\bar{R}_{t}+\sigma_{R_{t}} \varepsilon_{R_{t+1}}\right] \lambda_{t}+R_{t}^{f}\left(1-\lambda_{t}\right)\right\}+\left(\bar{y}_{t}+\sigma_{y_{t}} \varepsilon_{y_{t+1}}\right)
$$

and the household's problem (7) can be rewritten as:

$$
\begin{aligned}
H\left(w_{t}, \bar{R}_{t}, \bar{y}_{t}, \sigma_{R_{t}}, \sigma_{y_{t}}, R_{t}^{f} ; m^{-}\right) \\
=\operatorname{Max}_{c_{t}, \lambda_{t}} U\left(c_{t}, E_{t}\left[\phi \left(\left(w_{t}-c_{t}\right)\left[\left(\bar{R}_{t}+\sigma_{R_{t}} \varepsilon_{R_{t+1}}\right) \lambda_{t}+R_{t}^{f}\left(1-\lambda_{t}\right)\right]\right.\right.\right. \\
\left.\left.\left.\quad+\left(\bar{y}_{t}+\sigma_{y_{t}} \varepsilon_{y_{t+1}}\right) ; G_{e}\right)\right]\right)
\end{aligned}
$$

where $G_{e}$ is the distribution of the variables $\varepsilon_{R_{t+1}}, \varepsilon_{y_{t+1}}$ and $m^{-}$represents the moments of $G_{e}$.

Differentiating the indirect utility function $H$ and applying the Envelope Theorem, we get: 12

$$
\begin{aligned}
& \frac{\partial H(\cdot)}{\partial \bar{y}_{t}}=U_{2}(\cdot) E\left[\phi^{\prime}(\cdot)\right] \\
& \frac{\partial H(\cdot)}{\partial \bar{R}_{t}}=U_{2}(\cdot) E\left[\phi^{\prime}(\cdot)\right]\left(w_{t}-c_{t}\right) \lambda_{t} \\
& \frac{\partial H(\cdot)}{\partial R_{t}^{f}}=U_{2}(\cdot) E\left[\phi^{\prime}(\cdot)\right]\left(1-\lambda_{t}\right)\left(w_{t}-c_{t}\right)
\end{aligned}
$$

which can be used to obtain the consumption function and the demand for the market portfolio as:

$$
\begin{gathered}
c_{t}=w_{t}-\left[\frac{\partial H(\cdot)}{\partial \bar{R}_{t}}+\frac{\partial H(\cdot)}{\partial R_{t}^{f}}\right] / \frac{\partial H(\cdot)}{\partial \bar{y}_{t}} \\
\lambda_{t}=\frac{\partial H(\cdot)}{\partial \bar{R}_{t}} /\left[\frac{\partial H(\cdot)}{\partial \bar{R}_{t}}+\frac{\partial H(\cdot)}{\partial R_{t}^{f}}\right]
\end{gathered}
$$

Since we are interested in equilibrium consumption and asset demand, let us consider the implications of the economy's equilibrium conditions. In equilibrium, all markets clear and all prices, including $R_{t}^{f}$, are determined by the exogenous variables. ${ }^{13}$ Let the equilibrium risk free rate, consumption and asset demand be given by $R_{t}^{f *}=R_{t}^{f}\left(w_{t}, m_{t}\right), c_{t}^{*}=c_{t}^{*}\left(w_{t}, m_{t}\right)$ and $\lambda_{t}^{*}\left(w_{t}, m_{t}\right)$ respectively. Denote the derivatives of $H$, evaluated at the equilibrium, as:

\footnotetext{
$12>$ From the Theorem of the Maximum (Berg (1963)), it follows that the optimal solution for consumption is upper semicontinuous. If, in addition, it is unique, then indirect utility is differentiable. This is the standard result in consumer theory (see for example, Machina (1984)). Note that even if the utility function has kinks, the indirect utility function is differentiable (with upper semi continuity and uniqueness). For example, a Leontief utility function gives rise to a differentiable indirect utility function.

${ }^{13}$ See Appendix B.
} 


$$
\begin{aligned}
\left.\frac{\partial H(\cdot)}{\partial x}\right|_{c_{t}^{*}, \lambda_{t}^{*}, R_{t}^{f *}} & \equiv \frac{\partial H(*)}{\partial x} \\
\text { for all } x & =w_{t}, \bar{R}_{t}, \bar{y}_{t}, \sigma_{R_{t}}, \sigma_{y_{t}}, R_{t}^{f} ;
\end{aligned}
$$

Since in equilibrium, $\lambda_{t}^{*}=1$, it follows immediately from (24) that in equilibrium:

$$
\frac{\partial H(*)}{\partial R_{t}^{f}}=0
$$

In other words, in equilibrium, $R_{t}^{f}$ will not affect utility, since the net supply of bonds in the economy is zero.

The equilibrium consumption function is, therefore, given by:

$$
\begin{aligned}
c_{t}^{*} & =w_{t}-\frac{\partial H(*)}{\partial \bar{R}_{t}} / \frac{\partial H(*)}{\partial \bar{y}_{t}} \\
& \equiv c_{t}^{*}\left(w_{t}, \bar{R}_{t}, \bar{y}_{t}, \sigma_{R_{t}}, \sigma_{y_{t}}, ; m^{-}\right)
\end{aligned}
$$

Equilibrium consumption easily obtained from the ratio of marginal utilities of $\bar{R}_{t}$ and $\bar{y}_{t}$, as a "Roy's-Identity-like" equation. Furthermore, (26) is empirically tractable in terms of the moments of $R_{t+1}, y_{t+1}$ and wealth. In the next section we show how the indirect utility function can be used to obtain the equity premium function as well.

\subsection{Equity Premium and the Sharpe Ratio}

Expanding equation (9), we obtain the expression for the equity premium, $e p_{t}$, as:

$$
\begin{gathered}
e p_{t} \equiv E_{t}\left[R_{t+1}\right]-R_{t}^{f}=-\frac{\operatorname{cov}_{t}\left[\frac{\partial \phi}{\partial w_{t+1}}, R_{t+1}\right]}{E_{t}\left[\frac{\partial \phi}{\partial w_{t+1}}\right]} \\
=-\frac{\operatorname{cov}_{t}\left[\frac{\partial \phi}{\partial w_{t+1}}, \varepsilon_{R_{t+1}}\right]}{E_{t}\left[\frac{\partial \phi}{\partial w_{t+1}}\right]} \sigma_{R_{t}}
\end{gathered}
$$

Applying the Envelope Theorem to $H(\cdot)$, we obtain:

$$
\frac{\partial H(\cdot)}{\partial \sigma_{R_{t}}}=U_{2} E_{t}\left[\frac{\partial \phi}{\partial w_{t+1}} \cdot \varepsilon_{R_{t}}\right]\left(w_{t}-c_{t}\right) \lambda_{t}
$$

Evaluating this at the equilibrium and dividing by $\frac{\partial H(*)}{\partial \bar{R}_{t}}$ we get the equilibrium equity premium as: ${ }^{14}$ :

$$
\begin{aligned}
e p_{t}^{*} & =-\left[\frac{\partial H(*)}{\partial \sigma_{R_{t}}} / \frac{\partial H(*)}{\partial \bar{R}_{t}}\right] \sigma_{R_{t}} \\
& \equiv e p_{t}^{*}\left(w_{t}, \bar{R}_{t}, \bar{y}_{t}, \sigma_{R_{t}}, \sigma_{y_{t}}, ; m^{-}\right)
\end{aligned}
$$

\footnotetext{
${ }^{14}$ See Appendix A.II. for derivation.
} 
The equity premium can be easily related to the CAPM Sharpe ratio. Using (31), the Sharpe ratio $\left(s r_{t}\right)$ can be written as:

$$
s r_{t}^{*}=\frac{e p_{t}^{*}}{\sigma_{R_{t}}}=-\frac{\partial H(*)}{\partial \sigma_{R_{t}}} / \frac{\partial H(*)}{\partial \bar{R}_{t}}
$$

The Sharpe ratio, in our general equilibrium framework, turns out to be simply the slope of the indifference curve (in $\bar{R}_{t}, \sigma_{R_{t}}$ space), given by the ratio of marginal utilities of the standard deviation and mean return on market portfolio.

Equation (31) provides empirically tractable expressions for the equity premium. If we know, or can estimate the form of the indirect utility function (and given information on the exogenous variables), we can calculate the right hand side of (31). The attractive feature of our model is that it enables us to obtain theoretical expressions for both the consumption and equity premium, which are determined by the moments of the forcing processes (for $R_{t}$ and $\left.y_{t}\right)$ and the wealth. Furthermore, these expressions hold for any underlying preference structure, including non-expected utility.

It is useful to contrast our approach to the standard approach in the literature. Since we do not rely on a specific primal utility function, we do not encounter problems that often arise from the choice of a specific functional form for the preference. For example, we are immune to the empirical limitations of the standard constant relative risk aversion (CRRA) class of utility functions frequently used in the literature. ${ }^{15}$

Besides its empirical limitations, the CRRA utility function has theoretical limitations as well. Geweke (2001) shows that the existence of an expected utility function is fragile with respect to the change in the distribution function of the random variables. In our framework, the stochastic discount factor, $M_{t+1}$, is determined by the indirect utility function and the moments of the underlying forcing process. ${ }^{16}$ The only restriction is that the parameters of $H(\cdot)$ must satisfy the restrictions required by an indirect utility function. ${ }^{17}$

A standard problem with any "dual approach" is that it is not always possible to "go from the dual to the primal." As a result, it is not always possible to infer the exact properties of underlying preferences from the indirect utility function. This is not a major issue in our paper, because our objective is to study the equity premium, rather than the explicit properties of preferences. We are able to study the equity premium without using an explicit preference structure.

\footnotetext{
${ }^{15}$ One needs an inordinately high risk aversion parameter if one uses a standard primal utility function of a constant relative risk aversion (CRRA) type. For example, in the context of a widely popular power utility function $\frac{c^{1-\gamma}}{1-\gamma}$ with a lognormal processes for consumption and returns, it can be shown that the equity premium is given by: $\gamma \operatorname{Cov}_{t}\left(R_{t+1}, \frac{c_{t+1}}{c_{t}}\right)($ see Campbell, Lo and Mackinlay, 1997, p. 307). One requires a large value of the risk aversion parameter $\gamma$ to reproduce the observed equity premium. Epstein and Zin (1991) derive a flexible form power utility model which provides a useul form of the equity premium nesting the consumption CAPM and the traditional CAPM. Using this nested model, Epstein and Zin and others test the adequacy of consumption CAPM and find that CAPM predicts the equity premium better. However, their method again rests on a specific functional form and the assumption of homoskedastic returns. Our method uses general preferences and allows for heteroskedastic returns.

${ }^{16}$ Note that by using (11), (19) and the envelope property, we have $M_{t+1}=\frac{\partial H / \partial w_{t+1}}{\partial H / \partial w_{t}}$.

${ }^{17}$ The properties mentioned in section 2.2. It may be possible to obtain additional properties, but this requires further assumptions about the utility and aggregator functions, and the distributions of the random variables.
} 


\section{Empirical Application}

\subsection{Stochastic Processes}

In principle $R_{t}$ and $y_{t}$ are endogenous in the model and are, therefore, functions of the exogenous variables and wealth. Appendix B provides a fully specified general equilibrium model which describes how $R_{t}$ and $y_{t}$ are determined. Without knowing the specific functional forms for preference and technology, however, we cannot ascertain the exact reduced form processes for $\left\{R_{t}\right\}$ and $\left\{y_{t}\right\}$. Since neither our theoretical model, nor our empirical application use specific functional forms, we hypothesize these processes by the following simple AR form as an approximation. ${ }^{18}$

$$
\begin{gathered}
R_{t}=\gamma_{R}+\gamma_{R 1} R_{t-1}+\gamma_{R 2} R_{t-2}+e_{R t} \\
y_{t}=\gamma_{y}+\gamma_{y 1} y_{t-1}+\gamma_{y 2} y_{t-2}+e_{y t}
\end{gathered}
$$

where $e_{R}, e_{y}$ are distributed according to a bivariate normal distribution with $E\left(e_{y}\right)=$ $E\left(e_{R}\right)=0$ and a covariance matrix $S$, with $\operatorname{Var}\left(e_{y}\right)=\sigma_{y}^{2}, \operatorname{Var}\left(e_{R}\right)=\sigma_{R}^{2}$ and $\operatorname{Cov}\left(e_{R}, e_{y}\right)=$ $\sigma_{R y} \cdot{ }^{19}$ To obtain time varying values for the covariance matrix, the errors are assumed to follow the multivariate $\mathrm{ARCH}$ process given by: ${ }^{20}$

$$
\begin{gathered}
\sigma_{y t}^{2}=\beta_{y}+\beta_{y}^{1} e_{y t-1}^{2} \\
\sigma_{R t}^{2}=\beta_{R}+\beta_{R}^{1} e_{R}^{2} t-1
\end{gathered}
$$

A multivariate ARCH specification similar to the one in (35) has been introduced by Engle, Granger and Kraft (1984) and has been applied in several studies of financial markets ${ }^{21}$.

\subsection{Estimating Equity Premium and Consumption Functions}

We use a non-parametric technique to estimate the consumption and equity premium functions. The advantage of using this method is that we do not have to choose a functional form for the indirect utility function, since this method lets the data "choose" the form. Similarly, it frees us from having to make specific assumptions regrading the properties of the stochastic process (the error terms, $v_{c}(t), v_{e p}(t)$, which are defined below) in the consumption and equity premium functions.

Given the estimates of the moments, we estimate the consumption and actual equity premium functions nonparametrically. ${ }^{22}$ We rewrite the consumption and equity premium functions (26) and (31) as:

$$
c_{t}=c_{t}\left(w_{t}, \bar{R}_{t}, \bar{y}_{t}, \sigma_{R_{t}}, \sigma_{y_{t}} ; m^{-}\right)+v_{c}(t)
$$

\footnotetext{
${ }^{18}$ This approximation is not out of line with the theory. $\left\{R_{t}\right\}$ and $\left\{y_{t}\right\}$ would indeed display serial correlation because the capital stock carries over the shock from one period to another, which is a typical property of this class of models with Markovian decision rules.

${ }^{19}$ Note that the normal distribution is uniquely determined by the means and covariance matrix. Hence, in this case, a finite number of moments completely characterizes the distribution function $G$.

${ }^{20}$ In general we should also consider the correlation between $R$ and $y$ and include: $\sigma_{R y} t=\beta_{R y}+\beta_{R y}^{1} e_{R t-1} e_{y} t-1$. Examining the two series, we do not find any significant correlation $(\rho=.07)$. Thus, we did not include the covariance in the ARCH specification.

${ }^{21}$ See Bollerslev, Engle and Wooldridge (1988), Baillie and Myers (1991). See also Bera and Higgins (1993) for a survey of multivariate ARCH.

${ }^{22}$ For a discussion of nonparametric estimation see Pagan and Ullah (1999).
} 


$$
e p_{t}=e p_{t}\left(w_{t}, \bar{R}_{t}, \bar{y}_{t}, \sigma_{R_{t}}, \sigma_{y_{t}} ; m^{-}\right)+v_{e p}(t)
$$

where $v(t) \equiv\left\{v_{c}(t), v_{e p}(t)\right\}$ is the column vector of disturbances at time $t$.

We apply the following procedure to accommodate the fact that the data is for observed (actual) equity premium. We define the actual equity premium at time $t$, $a e p_{t}$, as:

$$
a e p_{t}=e p_{t}+\eta_{t}
$$

where $\eta_{t}$ is a white noise variable (with zero mean). The actual equity premium equation that we estimate is, then, written as:

$$
a e p_{t}=e p_{t}\left(w_{t}, \bar{R}_{t}, \bar{y}_{t}, \sigma_{R_{t}}, \sigma_{y_{t}} ; m^{-}\right)+\widetilde{v}_{e p}(t)
$$

where $\widetilde{v}_{e p}(t) \equiv v_{e p}(t)+\eta_{t}$.

We estimate the consumption and actual equity premium equations non-parametrically, as follows. Consider, for example, the random variable $X$ and a vector $z=\left[z_{1}, \ldots, z_{q}\right] .{ }^{23}$ The $a^{t h}$ order conditional moment of $X$ given $z$ is is $m_{a}(z)=E\left(X^{a} \mid z\right)$ for $a=1,2, \ldots$ We consider the nonparametric kernel estimation of $m_{a}(z)$. In general, for $a=1$, the kernel estimator is simply a weighted average

$$
\hat{m}_{1}(z)=\sum_{i=1}^{n} X_{i} w_{i}\left(z_{i}, z ; K\left(z_{i}, z, h_{z_{i}}\right)\right)
$$

where $h_{z_{i}}$ is the window width, or smoothing parameter, $K\left(z_{i}, z, h_{z_{i}}\right)$ is the Kernel function and the $w_{i}^{\prime} s$ are the weights. Intuitively, the estimator $\hat{m}_{1}(z)$ is the weighted average of the $X_{i}$ values corresponding to those $z_{i}^{\prime}$ s which are around $z$, the point at which $\hat{m}_{1}$ is calculated. The weights are given by the kernel function $K(\cdot)$ which is usually chosen to be a symmetric density around zero and is such that it gives a low weight to these observations $z_{i}$ that lie far from $z$. It is well known that the choice of kernel does not seem to matter a great deal (see, for example, Ullah (1988, P. 643)). The window width $h$ is an important parameter and its choice determines the 'size' of the interval around $z$, over which the observations are averaged. Usually, the larger the $h$ is, the less is the variance and the smoother the curve, but the larger the bias.

Note that $\hat{m}_{1}(z)$ is the sample estimate of the population average of $X$ values conditional on $z ; E(X \mid z)$. One can, therefore, write the nonparametric estimators of $m_{a}(z)$, the average of $X^{a}$ conditional on $z$, as ${ }^{24}$

$$
\hat{m}_{a}(z)=\sum_{i=1}^{n} X_{i}^{a} w_{i}
$$

Using this, we can write the nonparametric estimator of the second conditional moment around the mean as:

$$
\hat{\mu}_{2}(X \mid z)=\hat{m}_{2}(z)-\hat{m}_{1}^{2}(z)
$$

We use the programme "Portable Nonparametric Kernel Estimation version 1.7.1" for estimation. ${ }^{25}$ The programme option that we use applies the "local linear" method, it allows the data to choose the best band width and uses the Second Order Gaussian Kernel. ${ }^{26}$

\footnotetext{
${ }^{23}$ In our case $X$ is either $c_{t}$, or aept and the vector $z$ is given by: $w_{t}, \bar{R}_{t}, \bar{y}_{t}, \sigma_{R_{t+1}}, \sigma_{y_{t+1}}$.

${ }^{24}$ The asymptotic properties of $\hat{m}_{a}(z)$ are well established in the literature (See for example, Singh and Tracy (1977)).

${ }^{25}$ The programme was developed by Jeff Racine.

${ }^{26}$ Consider the function $E(X / z)$. Assume that it is second order differentiable. The linear approximation around $z_{0}$ is given
} 


\subsection{Data}

We apply the model to aggregate US data for the period 1929-2001. The data include the (time varying) moments of $R$ and $y$, wealth, consumption and the equity premium. The series for the additive shock, $y$, is measured by wages plus transfer payments. The series for the wealth, $w$, is measured by the year-end estimates of fixed assets. All these series are inflation adjusted to be consistent with the theoretical model. ${ }^{27}$

The series for actual equity premia is taken from Ibbotson and Associates (2002), where it is computed as the difference between large company stock returns and the T-bill rates (both inflation adjusted). To maintain consistency, our proxy for $R$ is the (same) large company stock return.

\subsection{Results}

Before we discuss the results, it is useful to compare our methodology with the common approach in the literature. In earlier work, the equity premium puzzle was examined by the calibration method, where one compares the summary statistics (e.g., mean equity premium) of the model with the data. Our approach enables us to estimate the consumption and equity premium functions using the entire historical series. We can, therefore, evaluate the extent to which the equity premium puzzle is solved, by evaluating the model's ability to explain observed consumption and equity premia.

First, we use the maximum likelihood technique to estimate equations (33) and (34) jointly, subject to the specification in (35). ${ }^{28}$ Using the parameter estimates, we obtain the estimates of the means and covariance matrix of $R$ and $y$. The parameter estimates for equations (33) - (35) are given in Table 1.

Given the estimated moments, we use the nonparametric technique described above to estimate the consumption and equity premium functions. The actual and nonparametric estimated values of equity premia and consumption are shown in Figures 1 and 2 respectively. Looking at these figures, we see that the nonparametric model is able to predict consumption very well. In addition, it also does reasonably well in predicting equity premia. The measure of "goodness of fit", which corresponds to the usual $R^{2}$ in a parametric model, is .9 for the consumption function and .67 for the equity premium function. ${ }^{29}$ The model captures the observed smooth consumption patterns and the high volatility and mean of equity premia during the sample period. The means and standard deviations of actual and predicted equity premia are $\{9.71 \%, 0.186\}$ and $\{9.89 \%, 0.130\}$, respectively.

Our results suggest that a preference structure which is general, both theoretically (based on the indirect utility function) and empirically (using a nonparametric technique that lets the data choose the functional form), can resolve the equity premium puzzle, at least, partially.

by: $E\left(X / z_{0}\right) \approx E(X / z)+(\partial E(X / z) / \partial z)^{\prime}\left(z_{0}-z\right) \equiv a+b^{\prime}\left(z_{0}-z\right)$. The estimation of the mean is the same as the estimation of the intercept. Hence, we choose $a$ and $b$ to minimize: $\sum\left(X_{i}-a-b^{\prime}\left(z-z_{i}\right)\right)^{2} K(\cdot)$, where $K(\cdot)$ is the kernel function used. The local linear method is described in Racine (2003) and is further discussed in Fan and Gijbels (1996). The scaling factors for $\bar{y}, \bar{R}_{t}, \sigma_{R_{t+1}}, \sigma_{y_{t+1}}$ and $w_{t}$ were $9.58,6.12, .519,1.41$ and .98 , respectively.

${ }^{27}$ Further details of data computation and sources are described in the Data Sources Appendix.

${ }^{28}$ In addition to the specification above we also tried a wide range of other possiblities, such as various GARCH models different AR orders, etc. The final results were similar in the sense that our conclusions regarding the consumption function and equity premia were not affected.

${ }^{29}$ For example, for the variable $X$, the goodness of fit measure is calculated as: $R^{2}=\rho_{x \widehat{x}}^{2}=\frac{\left[\sum_{i=1}^{n}\left(X_{i}-\bar{X}\right)\left(\widehat{X}_{i}-\bar{X}\right)\right]^{2}}{\sum_{i=1}^{n}\left(X_{i}-\bar{X}\right)^{2} \sum_{i=1}^{n}\left(\widehat{X}_{i}-\bar{X}\right)^{2}}$. This measure is identical to the traditional $R^{2}$ if the model is linear, with an intercept. 


\section{Conclusion}

In this paper, we provide a general and practically useful framework for the derivation of the consumption and equity premium functions. Applying duality in a dynamic context, we derive simple explicit expressions for consumption and equity premium functions. We show that these functions can be easily obtained by "Roy's Identity like" equations, from the marginal utilities of the indirect utility function. The advantage of our approach is that one can derive and estimate these functions for any underlying preferences, including nonexpected utility functionals. In our framework, the Sharpe Ratio is easily obtained within a general equilibrium setting and it resembles the standard result in a mean-variance portfolio model.

Our method provides estimable equations for equity premium and consumption functions. Applying a nonparametric technique, we estimate these two functions using US aggregate data. We find that the model does very well in explaining observed smooth consumption patterns. In addition, its ability to explain the observed high mean and volatility of equity premia is reasonable. These empirical results suggest that a general and flexible preference structure may be a promising approach to studying and solving the equity premium puzzle.

\section{Appendix A}

\section{First order conditions (8) and (9):}

(I.i) The first order condition for consumption is: $U_{1}-U_{2} E_{t}\left[\frac{\partial \phi}{\partial w_{t+1}} \widetilde{R}_{t+1}\right]=0$, where $U_{1} \equiv \frac{\partial U}{\partial c_{t}}$. Divide both sides by $U_{1}$ to get $U_{2} E_{t}\left[\frac{\frac{\partial \phi}{\partial w_{t+1}}}{U_{1}} \widetilde{R}_{t+1}\right]=1$. From the Envelope Theorem, we obtain that $\frac{\partial \phi}{\partial w_{t}}=U_{2} E_{t}\left[\frac{\partial \phi}{\partial w_{t+1}} \widetilde{R}_{t+1}\right]$. But, the first order condition implies that: $U_{2} E_{t}\left[\frac{\partial \phi}{\partial w_{t+1}} \widetilde{R}_{t+1}\right]=U_{1}$, and therefore, $\frac{\partial \phi}{\partial w_{t}}=U_{1}$. Plugging this into the first order condition, we get: $U_{2} E_{t}\left[\frac{\frac{\partial \phi}{\partial w_{t+1}}}{\frac{\partial \phi}{\partial w_{t}}} \widetilde{R}_{t+1}\right]=1$.

(I.ii) The first order conditions for $\lambda_{t}$ is: $U_{2} E_{t}\left[\frac{\partial \phi}{\partial w_{t+1}}\left(R_{t+1}-R_{t}^{f}\right)\left(w_{t}-c_{t}\right)\right]=0$. Dividing by $U_{2}\left(w_{t}-c_{t}\right)$, we get: $E_{t}\left[\frac{\partial \phi}{\partial w_{t+1}}\left(R_{t+1}-R_{t}^{f}\right)\right]$.

II. Equity Premium Function (31):

$U_{2} E_{t}\left[\frac{\partial \phi}{\partial w_{t}} \cdot \varepsilon_{R_{t}}\right]\left(w_{t}-c_{t}\right)=U_{2} \operatorname{Cov}\left(\frac{\partial \phi}{\partial w_{t}}, \varepsilon_{R_{t}}\right)\left(w_{t}-c_{t}\right)$.

Thus, $\frac{\partial H(*)}{\partial \sigma_{R_{t}}} / \frac{\partial H(*)}{\partial \bar{R}_{t}} \operatorname{Cov}\left(\frac{\partial \phi}{\partial w_{t+1}}, \varepsilon_{R_{t+1}}\right) / E_{t}\left[\frac{\partial \phi}{\partial w_{t+1}}\right]=-e p_{t} / \sigma_{R_{t}}$. Hence: $e p_{t}=-\frac{\partial H(*)}{\partial \sigma_{R_{t}}} / \frac{\partial H(*)}{\partial \bar{R}_{t}} \sigma_{R_{t}}$.

\section{Appendix B}

\subsection{A General Equilibrium Asset Pricing Model}

In this appendix we lay out a fully specified general equilibrium asset pricing model, which describes how $R_{t}$ and $y_{t}$ are endogenously determined. 


\section{Technology}

At date $t$, output, $q_{t}$, is produced with the predetermined capital stock, $k_{t-1}$ and labor, $l_{t}$, according to the constant returns to scale technology:

$$
q_{t}=\varepsilon_{t} F\left(k_{t-1}, l_{t}\right)
$$

where $\epsilon_{t}$ is a random productivity shocks dictated by nature.

\section{Households}

At date $t$, the representative household consumes, $c_{t}$, supplies a fixed amount of labor (normalized at unity) to the firm, at a competitive wage, $\psi_{t}$ and participates in share and bond markets. A share is an ownership claim to the capital stock in the economy. Define the stock of shares held by the household at date $t$ as $z_{t}$. At date $t$ the household buys net shares of, $z_{t}-z_{t-1}$ at a price $p_{t}$, in the stock market. Each share yields a dividend, $d_{t}$. The household also buys and sells bonds, $b_{t}$, at a price $p_{t}^{b}$, in the bond market. Such a bond pays one unit of sure consumption in the following period.

The household, thus, makes its decisions to maximize life time utility subject to the sequential budget constraint (1).

\section{Firms}

The representative firm owns all the capital stock and makes production and investment decisions. At each date $t$, after observing the productivity shock, $\varepsilon_{t}$ and given its (previous period) capital stock, $k_{t-1}$, a representative firm chooses its output, $q_{t}$, labour, $l_{t}$, and physical investment, $I_{t}$. The firm pays labour a competitive wage, $\psi_{t}$, and its capital stock is subject to a depreciation rate, $\delta$. The net cash flow, $q_{t}-\psi_{t} l_{t}-I_{t}$, is distributed to the household as dividend, $d_{t} \cdot{ }^{30}$

The representative firm, therefore, solves the following maximization problem:

$$
\begin{aligned}
& \max _{l_{t}, I_{t}, q_{t}}\left\{E_{0} \sum_{t=0}^{\infty}\left[q_{t}-\psi_{t} l_{t}-I_{t}\right] \prod_{i=1}^{t} \frac{1}{1+\rho_{i}}:\right. \\
k_{t}= & (1-\delta) k_{t-1}+I_{t}, \text { and }(43) \\
k_{0}= & \text { given. }
\end{aligned}
$$

where $\rho_{i}$ is the time varying discount rate facing the firm.

\section{Government}

The government finances a stream of spending, $G_{t}$, with lump-sum taxes, $T_{t}$, paid by the households. ${ }^{31}$ In other words, the government budget constraint is:

$$
G_{t}=T_{t}
$$

\footnotetext{
${ }^{30}$ This is a simplifying assumption, but as is well known, in this setting the firm's dividend policy does not affect the value of the firm (see Brock (1982).

${ }^{31}$ We assume that the government's spending does not enter into the household's utility function; nor does it serve any productive role in the economy.
} 


\section{Equilibrium}

The equilibrium for this economy is specified as follows:

(i) Facing $\left\{d_{t}\right\},\left\{\psi_{t}\right\},\left\{T_{t}\right\},\left\{p_{t}\right\},\left\{p_{t}^{b}\right\}$, the household chooses $\left\{c_{t}\right\},\left\{z_{t}\right\},\left\{b_{t}\right\}$ to maximize lifetime utility.

(ii) Facing $\left\{\varepsilon_{t}\right\},\left\{\psi_{t}\right\},\left\{\rho_{i}\right\}_{i=1}^{t}$, the firm chooses $\left\{I_{t}, l_{t}\right\}$ by solving problem (44).

(iii) The discount rate, $\rho_{t}$, facing the firm, must be consistent with the household's perceived marginal rate of substitution between $c_{t-1}$ and $c_{t}\left(M R S_{c_{t-1}, c_{t}}\right)$. In other words, $\frac{1}{1+\rho_{t}}=M R S_{c_{t-1}, c_{t}}$.

(iv) The government's budget constraint (46) holds.

(v) At each $t$, markets for stocks, bonds, labour and goods clear. This implies that: $z_{t}=1, b_{t}=0, l_{t}=1, q_{t}=c_{t}+I_{t}+G_{t}{ }^{32}$

In equilibrium, labor (which is 1 unit in supply) gets paid its marginal product which means:

$$
\psi_{t}=\epsilon_{t} F_{2}\left(k_{t-1}, 1\right)
$$

Moreover, we have the following arbitrage condition equating the stock return to investment return.

Lemma 1 The gross rate of return on stock at date $t, R_{t} \equiv \frac{p_{t}+d_{t}}{p_{t-1}}=1+\epsilon_{t} F_{1}\left(k_{t-1}, l_{t}\right)-\delta$.

Since by definition, stock is an ownership claim to the capital stock, in equilibrium, $p_{t}=k_{t}$. Using the fact that $d_{t}=q_{t}-\psi_{t}-I_{t}$ (see, (44)), one can rewrite the equilibrium gross return on stock (which we call $R_{t}$ hereafter) as:

$$
R_{t}=\frac{k_{t}+\epsilon_{t} F\left(k_{t-1}, l_{t}\right)-\psi_{t}-k_{t}+(1-\delta) k_{t-1}}{k_{t-1}}
$$

Finally, using the labor market equilibrium condition (47), we get $R_{t}=1+\epsilon_{t} F_{1}\left(k_{t-1}, l_{t}\right)-\delta$. Q.E.D.

In other words, in equilibrium $R_{t}, \psi_{t}$ are functions of $K_{t-1}$ and $\epsilon_{t}$.

\section{Data Sources}

The series for equity premia come from Ibbotson and Associates 2002 yearbook. Yearly data on personal income and its components as well as the CPI (US City Average, All Items) come from the Bureau of Labor Statistics Website (www.bls.gov) while annual GDP, fixed assets, and the implicit price deflator values are from the Bureau of Economic Analysis Website (www.bea.gov). All are in per capita values (using mid-year estimates of the US population), and deflated using the implicit price deflator. Data for wages and transfer payments come from the wages and salary disbursements component and the transfer payment component, respectively, of personal income. Both these series are deflated by the CPI and then added to generate a series for $y$. Fixed assets are year-end estimates of the current cost of private fixed assets. The series for consumption come from the personal consumption component.

\footnotetext{
${ }^{32}$ Equilibrium in the labor market means the labor (which is 1 unit in supply) gets paid its realized marginal product $\epsilon_{t} F_{2}\left(k_{t-1}, l_{t}\right)$. Since all households are identical, the net supply bonds $\left(b_{t}\right)$ is zero.
} 


\section{References}

Alvarez, F and U.J. Jermann, (2000). "Using Asset Prices to Measure the Cost of Business Cycles," Mimeo, University of Chicago.

Baillie, R.T. and R.J. Myers, (1991), "Bivariate GARCH estimation of Optimal Commodity Futures Hedge", Journal of Applied Econometrics, 16,109-124.

Bera, A.K. and M.L. Higgins, (1993), "Arch Models: Properties, Estimation and Testing", Journal of Economic Surveys, 7, 307-366.

Berg, C., (1963), Topological Spaces, tr. by Patterson, New York, MacMillan.

Bollerslev, T., R.F. Engle and J.M. Wooldridge, (1988), "A Capital Asset Pricing Model with Time-Varying Covariances", Journal of Political Economy, 96, 116-131.

Brock, W. (1982), "Asset Prices in a Production Economy," in: McCall J.J. ed, The Economics of Information and Uncertainty. University of Chicago press, Chicago. IL.

Campbell, J.Y, A. Lo, and C. Mackinlay, Econometrics of Financial Markets, Princeton University Press.

Cochrane J, and L. Hansen (1992), "Asset Pricing Explorations in Macroeconomics," in NBER Macroeconomics Annual 1992, MIT Press, Cambridge, 115-165.

Engle, R.F., C.W.J. Granger and D.F. Kraft, (1984), "Combining competing forecasts of infation using a bivariate ARCH model", Journal of Economic Dynamics and Control 8, pp. 151-165.

Epstein L.G. and S. E. Zin, (1991), "Substitution, Risk Aversion, and the Temporal Behavior of Consumption and Asset Returns: An Empirical Analysis", Journal of Political Economy, Vol. 99, 263-286.

Fan J. and I. Gijbels, (1996), Local Polynomial Modelling and Its Applications, Chapman and Hall, London.

Geweke, J., (2001), " A Note on Some Limitations of CRRA Utility," Economics Letters, 71, 341-345.

Hansen, L.P and R. Jagannathan (1991), "Implications of Security Market Data for Models of Dynamic Economies," Journal of Political Economy, 99, 225-62.

Ibbotson Associates (2002), Stocks, Bonds and Inflation-2002 Yearbook, Chicago: Ibbotson Associates Inc.

Jagannathan R, E.R. McGrattan and A. Scherbina, (2000), "The Declining U.S. Equity Premium", Federal Reserve Bank of Minneapolis Quarterly Review, Vol. 24, No. 4, pp. 3-19.

Kendall, M.G., (1969), The Advanced Theory of Statistics, Vol 1, Hafner publishing Company, New York.

Kocherlakota, N.R., (1996), "The Equity Premium: It's still a puzzle", Journal of Economic Literature, 34 (March), pp. 42-71.

Kreps, D.M and E.L. Porteus, (1978), "Temporal Resolution of Uncertainty and Dynamic Choice Theory", Econometrica, 46, 185-200.

Lettau, M and H. Uhlig, (2002) "Sharpe Ratio and Preferences: A Parametric Approach," Macroeconomic Dynamics, 6(2), 242-65.

Machina, M., (1984), "Temporal Risk and Induced Preferences", Journal of Economic Theory, 33, 199-231.

Mass-Collel, A., Whinston, M.D. and J.R. Green, (1995), Microeconomic Theory, Oxford University Press. 
Mehra R, and E. Prescott (1985), "The Equity Premium Puzzle," Journal of Monetary Economics, 24, 401-21.

Pagan, A. and Ullah, A. (1999), Nonparametric Econometrics, Cambridge University Press.

Racine, J., (2003), Portable Nonparametric Kernel Estimation version 1.7.1", Department of Economic, Syracuse University.

Reitz, T. (1988), "The Equity Risk Premium: A Solution?" Journal of Monetary Economics, 21, 117-132.

Singh, R.S. and D.S. Tracy, (1977), "Strongly Consistent Estimators of k-th Order Regression Curves and Rates of Convergence," Z.Wahrsch.Verw.Gebiete, 40, 339-348.

Treynor J.I. (1994), "The Invisible Cost of Trading," Journal of Portfolio Management, 21 (Fall), 71-78.

Ullah, A., (1988), "Non-Parametric Estimation of Econometric Functionals," Canadian Journal of Economics, 21, 3.

Watson, G.S., (1964), "Smoother Regression Analysis", Sankhya 26, 359-372.

Wilks, S.S, (1964), Mathematical Statistics, John Wiley \& Sons, New York. 


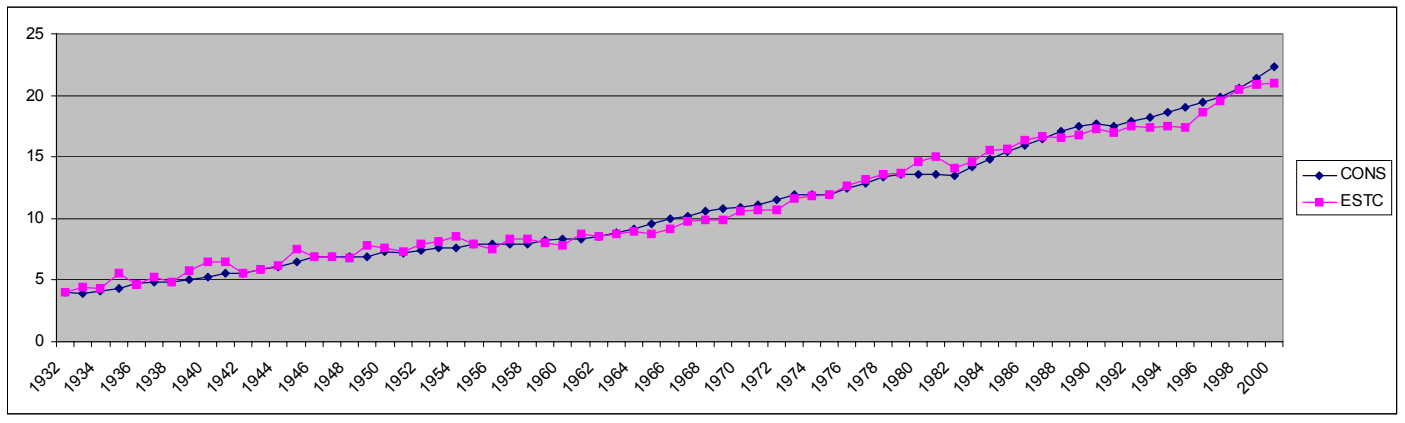

Figure 1: Actual and Estimated Consumption

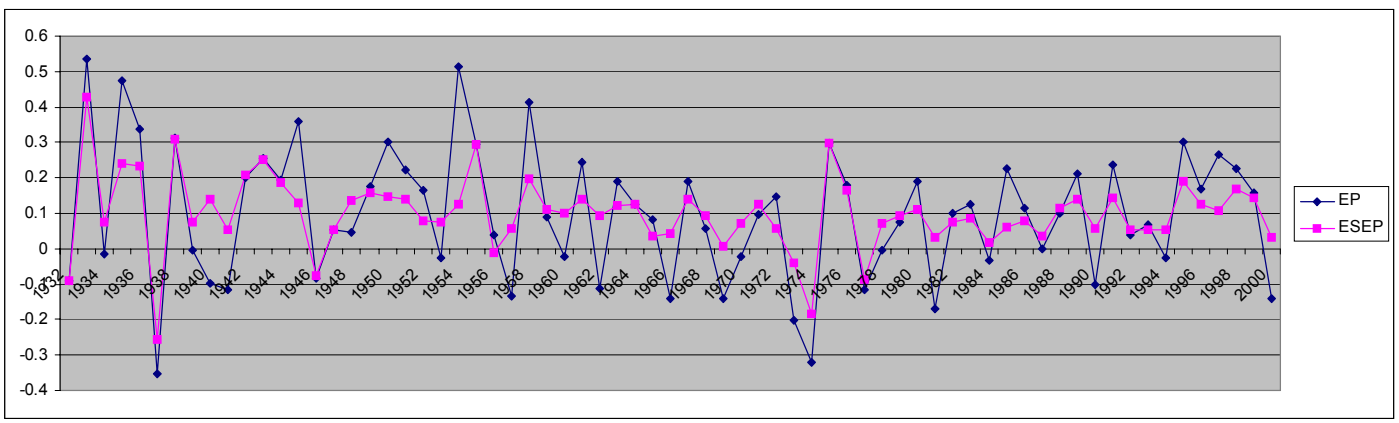

Figure 2: Actual and Estimated Equity Premia

Table 1: Maximum Likelihood Parameter Estimates of Income and Return Equations

\begin{tabular}{|l|l|l|}
\hline Parameter & Estimate & t- Statistic \\
\hline$\gamma_{R}$ & 1.45 & 8.22 \\
\hline$\gamma_{R 1}$ & -.15 & -1.12 \\
\hline$\gamma_{R 2}$ & -.16 & -1.37 \\
\hline$\gamma_{y}$ & .04 & .60 \\
\hline$\gamma_{y 1}$ & 1.52 & 8.70 \\
\hline$\gamma_{y 2}$ & -.51 & -2.84 \\
\hline$\beta_{R}$ & .17 & 5.89 \\
\hline$\beta_{R}^{1}$ & -.40 & -1.31 \\
\hline$\beta_{y}$ & .21 & 5.82 \\
\hline$\beta_{y}^{1}$ & .61 & 2.97 \\
\hline
\end{tabular}

